\title{
A New Route to Generating Black Holes
}

\author{
Observations indicate that a supernova of a "Wolf-Rayet" star produced a \\ black hole-suggesting that the class of black hole progenitors is larger \\ than was thought.
}

By Katherine Wright

$\Gamma$ or decades, astronomers have been scouring the skies in vain for signals of the explosions of a quirky type of star known as a Wolf-Rayet star. These stars have unusual spectra that are poor in hydrogen and rich in carbon, oxygen, and helium. The lack of evidence for a Wolf-Rayet supernova has led some in the field to surmise that, at the end of their lives, Wolf-Rayet stars do not explode but instead transform invisibly into black holes. Now, Avishay Gal-Yam of the Weizmann Institute of Science, Israel, and his colleagues have turned that idea on its head. The team has reported the

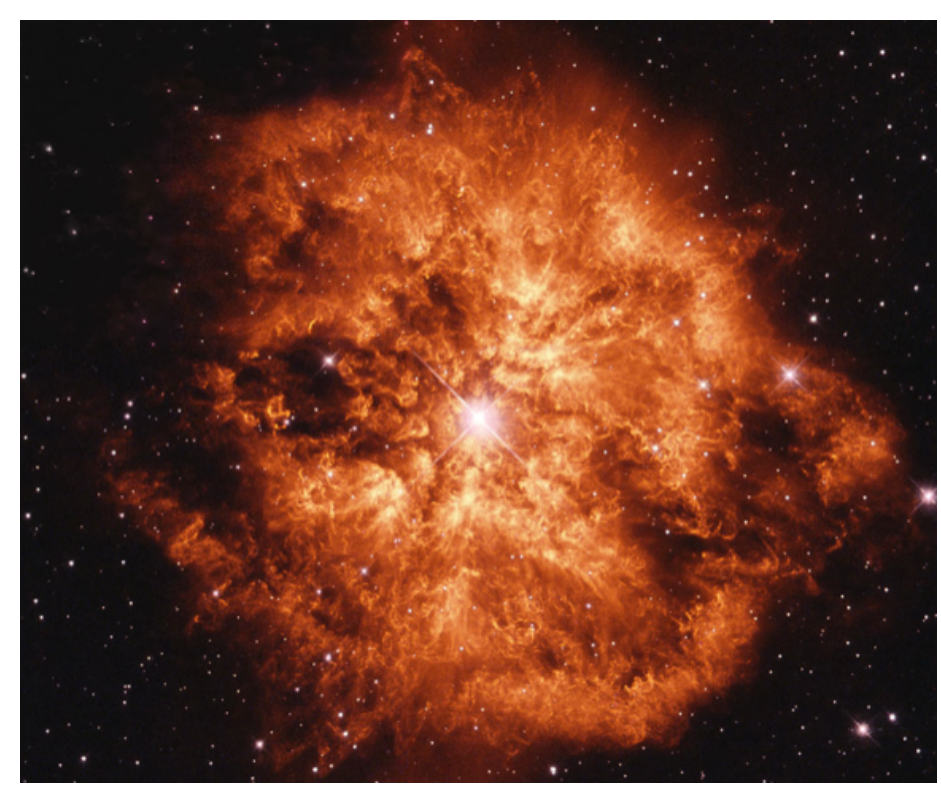

A Wolf-Rayet star and its surrounding nebula.

Credit: NASA/Hubble Space Telescope observation of a supernova on June 8, 2019, that appears to match the expectations for the explosion of a Wolf-Rayet star. The data also indicate that, after the explosion, the remnant of the star transformed into a black hole, a finding that requires physicists to update models of black hole progenitors.

Roughly every second, one of the observable Universe's stars dies in a fiery explosion. Despite the prevalence of these events, many questions remain about why, when, and how they happen. To gain insight into these issues, astronomers need information about both the supernova and the original star. But gaining information about the latter has proved tricky. A supernova provides a bright flash that can illuminate material in and around the star. But a supernova also synthesizes new materials, masking the star's original composition. "It's a conundrum," Gal-Yam says. "You can study the star because of the supernova, but the star is already destroyed."

In the late 2000s, researchers solved that problem by using faster detection of supernovae. Previously, images of the sky were taken once a month. That meant that, on average, a supernova was only detectable about two weeks after it commenced, long enough to obliterate any trace of the original star. But astronomers changed the image repetition time to one day. That timescale is short enough to capture information about the star's original composition and thus identify the progenitor of the supernova, Gal-Yam says. "We could finally see what distant stars are made of."

Using this technique, Gal-Yam and his colleagues have scoured the skies for interesting stars, in particular, Wolf-Rayet stars. 
These stars are relatively small, like our Sun, but about 10 times more massive. The winds that emanate from these bright stars blow at fantastic speeds, stripping away the stars' outer hydrogen and helium layers and revealing their inner heavier elements. To better understand how these stars evolve, researchers would like to detect a Wolf-Rayet explosion, something Gal-Yam and his colleagues have now achieved.

The team spotted supernova "2019hgp" a little more than a day after it exploded. Its spectra showed prominent lines for carbon, oxygen, and neon but not for hydrogen or helium. Analysis of the Doppler shifts of the lines indicated that the detected material was moving at speeds greater than 1500 $\mathrm{km} / \mathrm{s}$. (Our Sun's winds move at about $500 \mathrm{~km} / \mathrm{s}$ and those of supergiant stars, which can have the same mass as Wolf-Rayet stars, at about $10 \mathrm{~km} / \mathrm{s}$.) The data all indicate the explosion of a massive but compact star that contained the elements expected for a Wolf-Rayet star, Gal-Yam says.

"It's definitely an exciting result," says Paul Crowther, an astrophysicist at the University of Sheffield, UK. He notes that there are a few oddities about the observations. "We still expect some helium to be present," he says, and the rate of mass loss is much higher than expected for a Wolf-Rayet star. Still, he says it is "credible" that the observed event was a Wolf-Rayet explosion.

Besides indicating that Wolf-Rayet stars can explode in supernovae, the data have other implications for the understanding of the life cycle of these stars. Gal-Yam and his colleagues estimate that only about one tenth of the mass of the star was ejected during the explosion, leaving most of the star's 10 solar masses behind. That remaining mass is too large for the remnant to have formed a neutron star, as both theory and observations limit neutron stars to less than 2-3 solar masses. The mass remnant instead fits with that expected for a small black hole.

A black hole remnant is surprising, as models suggest that all "successful" supernovae-those that radiate above a certain threshold energy-leave behind neutron stars, Gal-Yam says. If some fraction of supernovae produce black holes, then black holes might be significantly more abundant than previously thought. Additionally, some Wolf-Rayet stars have the right mass such that their supernovae could provide the missing origin for the low-mass black holes detected by the gravitational-wave observatories LIGO and Virgo. "The result clearly has implications for our understanding of the number of black holes in the Universe," Gal-Yam says.

Katherine Wright is the Deputy Editor of Physics. 